\title{
EFFECT OF PHYSICAL CHANGES IN AGING ON DRIVING PERFORMANCE
}

\author{
Hideaki Nemoto, Takayuki Yanagishima and Mitsuru Taguchi \\ Vehicle Research Laboratory \\ Nissan Research Center \\ Nissan Motor Co.,Ltd. \\ Yokosuka City, Kanagawa JAPAN \\ E-mail: h-nemo@mail.nissan.co.jp
}

\begin{abstract}
Summary: The growing population of older drivers has led to societal demands for the development of technologies better suited to the characteristics of older people. One effective way of addressing this situation is to examine closely the structure that causes the performance of older drivers and then use that information to develop technologies. As the first step toward understanding that structure in this research, an attempt was made to identify significant elements of the structure from a human factors standpoint in order to pursue this approach efficiently. A questionnaire survey, an observation of driving behavior and an interview survey were conducted with older drivers. The most significant perspectives on driving were extracted from the results. Tests were then conducted with a driving simulator to verify those perspectives. Based on the surveys and tests, two significant characteristics were identified concerning the influence of aging on driving behavior. One concerns the disintegration of the stimulus-perception-cognition-response system and the other concerns the transition in attention.
\end{abstract}

\section{INTRODUCTION}

The growing number of older drivers has prompted societal demands for the development of technologies better adapted to the characteristics of older people in order to strike an optimum balance between public safety in the traffic environment and quality of life for senior citizens. In view of this situation, vehicle manufacturers need to consider the provision of technologies based on a universal design concept as much as possible, while keeping in mind the relationship between manufacturing cost and market acceptance. It is necessary to develop technologies that will also benefit younger users in addition to older people.

One approach to developing technologies beneficial to both age groups would be to probe the structure of the phenomena that cause differences between older and younger users when they are driving a vehicle. Based on that information, technologies beneficial to both age groups could be provided as one method of dealing with such differences. This approach seeks to make technological innovations by going back to the origins of engineering concepts and has the potential to accommodate the characteristics of a wide range of user segments.

At the initial stage of this approach, an effort was made from a structural viewpoint to gain insights into driving behavior characteristics that appear with increasing age in order to identify the perspectives of major importance. A questionnaire survey, an observation of driving behavior, 
an interview survey and a driving simulator tests were used in trying to gain insights from a structural standpoint. This procedure and the results obtained are explained in detail below.

\section{OVERVIEW OF SURVEYS AND RESULTS}

\section{Questionnaire Survey and Observation of Driving Behavior}

Description. A questionnaire survey about driving in general was conducted among a large number of older drivers (aged 60-78) in order to gather information on the characteristics of driving-related matters of strong concern to them.

To ascertain the specific details that the questionnaire survey did not reveal about the phenomena involved, observations were then made of the driving behavior of eight older drivers (aged 6575) by having an observer ride with them in their vehicles.

Results. The following typical findings were obtained as a result of tabulating the questionnaire responses and observation.

1. Questionnaire survey: items about which respondents felt increasingly unsafe or insecure

(1) Difficulty of driving at night (dazzling glare of the headlamps of oncoming vehicles, dimness of the headlamps of one's own vehicle)

(2) Reduced perception of one's own vehicle (partiality for certain driving behavior, narrow roads, backing into the garage, making 90-degree right turns, headway distance to a preceding vehicle

(3) Failure to confirm safety (failing to notice traffic signals, forgetting to stop momentarily, forgetting to check both ways)

2. Observation of driving behavior:

(1) Unstable driving operations

Their steering or pedal operations were jerky at times.

The vehicle's lateral position in its lane was not stable.

When parking, they were not able to position the vehicle as they intended.

(2) Missing or misperceived visual information depending on the circumstances

A pedestrian crossing the street at an intersection was nearly struck because attention was not paid to the pedestrian's behavior.

Traffic signals were mistakenly recognized.

(3) Failure to execute driving operations depending on the circumstances

The number of times the subjects braked to slow down near intersections decreased markedly.

Discussion. Looking at the survey results concerning the feeling of being unsafe or insecure, which is closely related to public safety in the traffic environment, it is clear that older drivers are greatly affected by and are highly concerned about their declining vision when operating a vehicle. Difficulty in obtaining visual information, signifies there is increased resistance to the acquisition of information visually. Accordingly, it is hypothesized that older people with deteriorated vision have to allocate greater attention to compensate for their insufficient visual 
faculty in order to obtain the same amount of visual information as an ordinary person with normal vision.

In this survey to observe driving behavior, there were also times when driving operations were not sufficiently executed, as typified by a reduced frequency of braking to decelerate the vehicle depending on the circumstances. One of the principal points noticed in this survey was the observation of a similar type of behavior in that the subjects not only failed to obtain visual information, they also failed to execute certain driving operations depending on the circumstances. This failure to recognize visual information or to perform driving tasks was observed near intersections where the driver's workload is presumed to be relatively high.

From this observation, it is hypothesized that allocating more attention as a means of compensation is a comprehensive characteristic that pertains not only to visual information but also to other driving actions as well. This is the hypothesis formulated about attention.

\section{Interview Survey}

Description. The eight subjects were also interviewed about their driving habits in addition to observing their driving behavior. The objective was to learn about their driving behavior in driving environments other than the ones observed in the survey. Interviews were conducted in face-to-face meetings.

Results. The following typical driving habits were identified on the basis of the interviews. - The subjects forgo driving in rainy weather and at night.

- They avoid intersections where right turns can be dangerous.

- They sit with the seatback in an upright position, making it easier to apply force to the pedals.

- They drive only on set routes where they are familiar with the roads.

Discussion. The results of the interviews revealed that the subjects deal with their diminished physical abilities (e.g., deteriorated vision) due to increasing age on a different dimension from their physical faculties. For example, they change their driving strategy, such as by refraining from driving in rainy weather and at night. Accordingly, it is thought that one approach to understanding this characteristic relationship seen for older drivers is to model the structure of driving actions (i.e., driving skill) hierarchically. This is the hypothesis formulated about driving skill. When driving actions are treated in a hierarchical structure, it is assumed that drivers endeavor to recognize and acquire skill in executing driving operations as a behavioral set at the highest possible level. The acquisition of driving skill is regarded as a process of integrating individual skills into a set of actions learned as a whole. This skill set incorporates a sequence of actions related to driving, i.e., perception, cognition, judgment and execution.

\section{Relationship between attention and driving skill}

The act of allocating attention to one area of driving skill indicates that an object of cognition separate from overall driving skill arises in a person's mind. When driving skill is regarded as a hierarchical structure, it is thought that drivers are cognizant of the individual actions composing driving skill, which is different from their cognizance of driving skill as an integrated whole. In 
allocating attention to the execution of those separate actions, it is necessary to address each of them individually. In order to process the overall task and an individual task in a short period of time, attention must be alternately paid to the two tasks. The need to allocate attention to a task to compensate for a decline in physical faculties can be expected to influence a person's performance in executing the overall task, in addition to that involving some diminished physical capacity.

\section{Driving Simulator Tests}

Description. Tests were conducted with a driving simulator to confirm quantitatively the aforementioned relationship between attention and driving skill. The test subjects were three older drivers and two younger drivers.

Test procedure. The test simulated a lane-keeping situation where a driver is following a preceding vehicle on an expressway. In order to vary the steering workload, the expressway course simulated on the driving simulator combined straight segments with three types of curves having a radius of 900, 600 and $300 \mathrm{~m}$, respectively. This difference in the radius of curvature varied the steering workload required to keep the vehicle in its lane. To keep the pedal operation workload at a constant level, the subjects were instructed to follow the vehicle ahead. The speed of the preceding vehicle was set at a constant $115 \mathrm{~km} / \mathrm{h}$. Under this condition, an investigation was made of whether the different steering workload levels due to the different radii of curvature affected the test subjects' operation of the accelerator pedal or not.

Method of analysis. Smoothness of operation was quantified in order to quantify the subjects' performance in operating the steering wheel and the accelerator pedal. It is assumed that when performance declines, smoothness of operation also decreases.

First, to calculate steering smoothness, prediction error e(n) is calculated from a time-series history of the steering angle signal sampled at 50-ms intervals using the equation noted below. We then focus on a certain time $n$. Using the steering angles of the previous three times (n-3, n-2, and n-1), we perform a second-order Taylor expansion centering on the time $n-1$. This operation yields the difference between the predicted steering angle $\theta \mathrm{p}(\mathrm{n})$ at time $\mathrm{n}$ (i.e., the steering angle likely to be obtained if the driver steers very smoothly) and the actual steering angle $\theta(n)$ at time $\mathrm{n}$. The prediction error $\mathrm{e}(\mathrm{n})$ at time $\mathrm{n}$ is thus obtained as a result. It is assumed that steering smoothness increases as the value of e(n) decreases.

$$
\begin{aligned}
e(n)= & \theta(n)-\theta_{\theta} P(n) \\
& \text { where } \theta P(N)=\theta(n+1)+(\theta(n-1)-\theta(n-2))+1 / 2((\theta(n-1)-\theta(n-2))-(\theta(n-2)-\theta(n-3)))
\end{aligned}
$$

The same procedure is used to determine the smoothness of accelerator pedal operation. Letting $d(n)$ denote the prediction error of accelerator pedal operation at time $n$, the following calculation is performed to judge the linkage between the variation of the two values e(n) and $d(n)$.

$$
\mathrm{C}=(\Sigma|\mathrm{e}(\mathrm{n})-\mathrm{e}| \bullet|\mathrm{d}(\mathrm{n})-\mathrm{d}|) /(\mathrm{N}-1)
$$

Where "e" is average value of $e(n)$, “d" is average value of $d(n)$ and " $N$ " is number of data. This operation calculates the sample covariance using absolute values. Covariance is generally used as 
an index showing the magnitude of dispersion and the direction of the relationship (direction of dispersion) of the data for two variables. The calculation was performed in this study with absolute values because the relationship between the magnitude of e(n) and d(n) was the only focus of concern.

Results. The test results are shown in Figs. 1-2. Figure 1 shows the magnitude of the steering perturbation on each curve relative to the straight segments as the baseline. These values were calculated with the steering angle entropy method (Boer, 1997). The results in Fig. 1 show that the increase in steering workload was larger for the older drivers than the younger ones.

Figure 2 shows the degree of interference between steering and accelerator pedal operation on each curve in relation to the index for the straight segments as a baseline. These values were calculated with the above-mentioned index equation (2). It is seen that the values of the index increase with an increasing steering workload. The younger drivers did not show any interference between steering and accelerator pedal operation even on the larger curves, but the degree of interference increased for the older drivers.

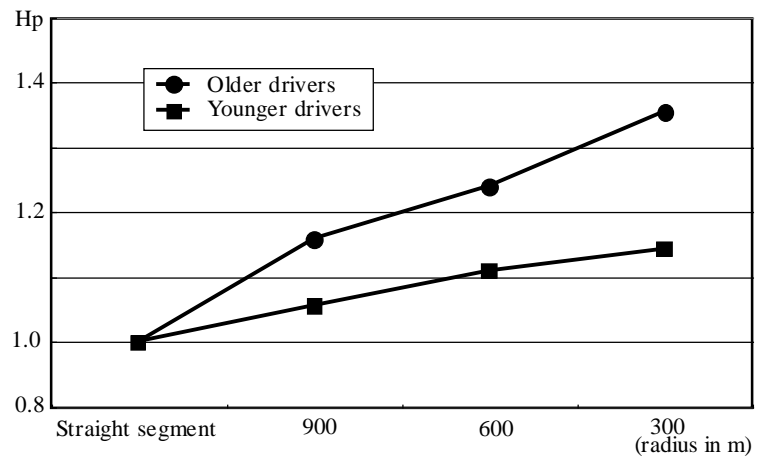

Fig. 1 Steering perturbation for each radius of curvature

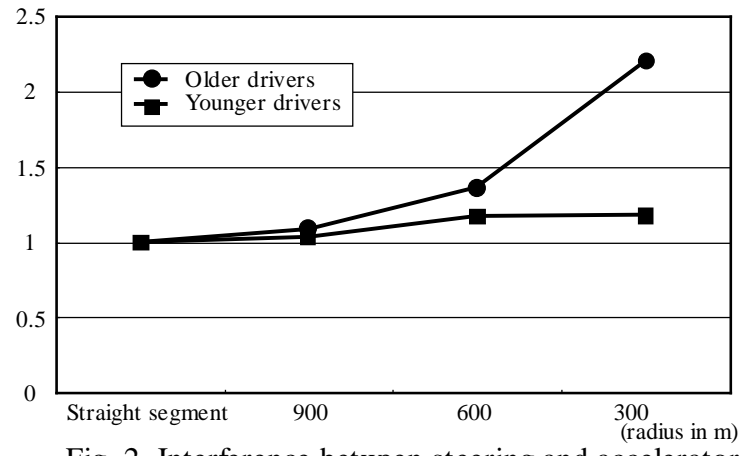

Fig. 2 Interference between steering and accelerator pedal operation for each radius of curvature

Discussion. It is known from experience that drivers are not particularly conscious of executing certain tasks, i.e., steering and pedal operations, among the driving actions for keeping a vehicle in its lane. Drivers are assumed to learn how to perform these tasks as an integrated driving skill set in accordance. When some particular driving task workload is increased under a lane-keeping situation, greater attention is paid to that task in proportion to the magnitude of the load. As a result, the driver perceives that particular operating task and the other tasks involved in keeping the vehicle in its lane as being separate tasks. It was observed that driving skill disintegrated for the older drivers and that steering interfered with their performance in operating the accelerator pedal.

Since a workload is determined by the relative relationship between the task and ability, it is dependent on the individual's ability. The number of multiple tasks that can be executed varies continuously according to the workload level, which means that the level of attention allocated changes the number of multiple tasks a person can handle. From this observation, it can be hypothesized that the level of attention allocated influences how easily a person can switch from one task to another.

\section{CONCLUSIONS}


Based on the results of the surveys and tests, two characteristics were identified as being highly important in understanding the effects of aging on driving performance.

(1) Integration and disintegration of the stimulus-cognition-response system

Older drivers concentrate their attention on an area of their driving skill in order to compensate for a decline in their physical faculties due to aging. To older drivers, this means that another task occurs that is separate from the driving actions they used to perform as one integrated skill set. An increased workload in a particular area of their driving skill due to diminished physical faculties has the effect of disintegrating their driving skill.

(2) Transition in attention

The execution of a given task in a short time through the application of a corresponding skill set and a segregated skill requires that attention be switched among multiple skills. The ease of switching attention is influenced by the extent to which attention is concentrated.

\section{REFERENCES}

Boer, E. R. and Liu. A. (Eds.), Cambridge Basic Research 1997 Annual Report, CBR TR 97-7, Cambridge Basic Research, Cambridge Massachusetts, 1997.

Korteling, J.E. Effects of skill integration and perceptual competition on age related differences in dual-task performance. Human Factors, 33, 35-44, 1991. 